\title{
Die Denkerin der Stunde? Neue Schriften von und über Hannah Arendt
}

\author{
Thomas Meyer
}

Online publiziert: 27. April 2021

(C) Der/die Autor(en) 2021

Zusammenfassung Das weltweit gestiegene Interesse an Leben und Werk Hannah Arendts hat in den letzten zehn Jahren eine unüberschaubare Menge an neuer Literatur hervorgebracht. Der Literaturbericht konzentriert sich zum einen auf Schriften Arendts, die erstmals zugänglich gemacht wurden. Ein Schwerpunkt liegt hier auf der neuen kritischen Edition ihrer Schriften. Zudem werden neuere Einführungen und Sekundärliteratur aus den Bereichen Literatur und Recht eingehender analysiert.

Schlüsselwörter Politische Theorie · Kritische Edition · Einführungen · Literatur · Recht

\section{The Thinker of the Hour? New Writings by and About Hannah Arendt}

Abstract The worldwide increased interest in the life and work of Hannah Arendt has produced a vast amount of new literature over the past 10 years. This literature survey focuses on Arendt's writings that have been made accessible for the first time, as well as on the new critical edition of her works. In addition, more recent introductions and secondary literature from the fields of literature and law are analyzed in detail.

Keywords Political Theory $\cdot$ Critical edition · Introductions $\cdot$ Literature $\cdot$ Law

T. Meyer $(\triangle)$

Philosophie Department, LMU München, München, Deutschland

E-Mail: thomas.meyer@1rz.uni-muenchen.de 


\section{Einführung}

Dreißig Jahre nach Wolfgang Heuers noch heute wichtigem Literaturbericht „Hannah Arendt - Politische Wissenschaft aus dem Blickwinkel von Minderheiten“ in dieser Zeitschrift (Heuer 1990), hat sich nahezu alles verändert. 1990 war es noch möglich eine nahezu vollständige Bibliografie der nach Arendts Tod 1975 erschienenen Sekundärliteratur anzugeben, sie auf wenigen Seiten zu besprechen und Desiderate aufzuzeigen. Bereits fünf Jahre später musste sich Harald Bluhm auf eine sehr eingeschränkte Rezeptionslinie beziehen, um die gestellte Aufgabe eines Literaturüberblickes noch leisten zu können (Bluhm 1995). Vier Jahre später legt die Philosophin Teresa Orozco eine ebenso prononcierte, wie kritische Literaturdiskussion zu Arendts ,Diagnose über den Verfall des Politischen und ihrer Absage an die politische Lösbarkeit der ,sozialen Frage““ vor (Orozco 1999, S. 95-110).

Die Gründe dafür, warum sich in Bezug auf Arendt nahezu alles verändert hat, liegen auf der Hand: die dramatischen Veränderungen der Weltlage in den Jahren 1989 und 2001, die von den USA geführten Kriege, das als Globalisierung benannte Phänomen einer eng vernetzten und damit voneinander abhängigen Welt, das Erstarken autoritärer Systeme, die Wahl Donald Trumps 2016, die fortdauernde Krise des Nationalstaaten-Konzept in eins mit den Fragilitäten internationaler Staatengemeinschaften, die Aushöhlungen traditioneller liberaler Demokratien und die Frage nach den Konsequenzen eines ungezügelten Kapitalismus lenkten den Blick auf bislang weniger beachtete Antworten aus der politischen Wissenschaft. Hinzu kam, dass die genannten Entwicklungen nationale wie internationale Rechtsrahmen veränderten und in der Folge deren ursprüngliche Begründungen nicht länger gültig erscheinen ließen. Und so rückte mit Hannah Arendt eine Denkerin in den Mittelpunkt der Aufmerksamkeit, die klassische Unterscheidungen wie Theorie und Praxis, Einzelnem und Staat hinter sich lässt und dazu Analysen en gros und en détail anbietet, dabei Texte von der Antike bis zu ihrer Gegenwart heranzieht, um ihre Erklärungen zu belegen. Insbesondere die bisherigen Konzeptionen universaler Menschenrechte wurden mittels Arendts Formulierung vom „Recht, Rechte zu haben“ weltweit neu oder anders rezipiert (DeGooyer et al. 2018).

Dieser kursorische Überblick sollte genügen, um die wichtigsten Anstöße und Felder zu benennen, die Arendts Schriften rund um den Globus eine hohe Attraktivität verschafften. Im Zuge der Rezeption entdeckte man dann ihre „Anschlussfähigkeit“: Konservative, liberale, linke und radikaldemokratische Ansätze berufen sich auf Arendts Schriften und offerieren dabei extrem gegensätzliche Lesarten. Und nicht wenige andere Autorinnen und Autoren profilierten sich gerade durch die entschiedene Absetzung von Arendt: Giorgio Agamben, Judith Butler und Slavoj Žižek, um nur die prominentesten zu nennen.

Das Interesse an Arendts Werk hatte sich noch nie auf die Akademie beschränkt, da sie selbst nie eine solche Beschränkung beförderte oder gar wollte. Seit ihrer Dissertation 1929 bei Karl Jaspers in Heidelberg über Der Liebesbegriff bei Augustin veröffentlichte sie keinen Text mehr, der sich direkt an ein ausschließlich akademisches Publikum wendete. Arendt schrieb bereits vor ihrer Flucht aus Deutschland 1933 Feuilletons, arbeitete ab 1941 für die Wochenzeitung Aufbau in New York, war in der Folge Gast in Radiosendungen und hinterließ im Laufe der Jahrzehnte nicht 
wenige Beiträge und Interviews für dieses Medium, darin allenfalls unter Philosophen von Theodor W. Adorno im deutschsprachigen Raum zahlenmäßig übertroffen.

Zudem gilt: Arendts Bekanntheit in deutschsprachigen Ländern war wesentlich durch ihre Vorträge möglich geworden, die ihren Reiz nicht zuletzt aus der Verschränkung von bildungsbürgerlicher Attitüde und unbedingt-schonungsloser Analyse bezogen, wenn man den vielen Berichten über ihre Auftritte Glauben schenken darf. Exemplarisch stehen dafür die Laudatio anlässlich der Verleihung des Friedenspreises des Deutschen Buchhandels an Karl Jaspers (1958) und die Rede bei der Überreichung des Lessing-Preises in Bremen ein Jahr später. Jaspers' und Arendts Reden in der Frankfurter Paulskirche waren so populär, dass die Firma AEG-Elotherm auf eigene Kosten einen Sonderdruck herstellen und an die Mitarbeiter als Weihnachtsgabe verteilen ließ. ${ }^{1}$

So verwundert es nicht, dass sie in den USA und in Europa stets auch als ,public intellectual" wahrgenommen wurde. Obwohl ihre Schriften regelmäßig und zumeist ausführlich in wissenschaftlichen Zeitschriften besprochen wurden, sie Forschungsaufenthalte und Gastprofessuren an renommierten Universitäten wie Berkeley, Princeton und Chicago hatte, bis sie schließlich1967 eine Dauerstelle an der New School of Social Research in New York annahm, lief die Rezeption zumeist über die sogenannten Massenmedien, also in Zeitungen und im Radio (Maffeis 2018). Als öffentliche, politisch intervenierende Intellektuelle wird Arendt bis heute in breiten Leserschichten wahrgenommen und als solche geschätzt, ja verehrt (Bernstein 2020). Phänomene, die sich nicht zuletzt in der ein gänzlich uneinheitliches Bild abgebenden Sekundärliteratur niedergeschlagen haben. Nicht nur aus Marketinggründen bieten Verlage Comics (hier herausragend: Krimstein 2018) oder Biografien für Jugendliche an, von denen insbesondere die von Alois Prinz (Prinz 2012) erfolgreich ist. Doch während z. B. die Ausstellung im Deutschen Historischen Museum (2020) sehr bewusst auf eine Vermittlung von Person und Werk gesetzt hatte, wartete der Katalog mit wissenschaftlicher Expertise auf (Boll et al. 2020).

Arendts Schriften sind zum Teil in einem Maße ,übersetzbar“, wie es bei keinem anderen Denker von Rang im 20. Jahrhundert möglich ist. Das haben zahlreiche Medien erkannt und nutzen diese Erkenntnis, die wiederum auf die wissenschaftliche Beschäftigung Auswirkungen hat, wie wir sehen werden.

Gänzlich verändert hat sich in den letzten Jahren vor allem die Quellenlage. Neben der nahezu vollständigen Digitalisierung von Arendts Nachlass in der Library of Congress in Washington DC und der freien Zurverfügungstellung zahlreicher Texte und Briefe im Internet, ist etwa durch die kritische Ausgabe von Arendts Werken völlig unbekanntes Material zugänglich geworden, das sich teils in Archiven, teils in privaten Händen befand. Selbst die ganz konventionelle Archivforschung, über Jahrzehnte keine Stärke der Interessierten, hat nun endlich auch in Sachen

\footnotetext{
${ }^{1}$ Ein entsprechendes Exemplar befindet sich im Besitz des Verfassers. Über solche und ähnliche Sonderdrucke ist bislang in der Arendt-Forschung nichts bekannt.
} 
Arendt Einzug gehalten. ${ }^{2}$ Davon zeugen zahlreiche, noch genauer zu betrachtende Publikationen.

In Deutschland haben vornehmlich Forscherinnen die zuletzt beschriebene Entwicklung konstruktiv genutzt: Zu nennen wären hier vor allem die abseits von der Universität agierenden Marie Louise Knott, Ursula Ludz und Ingeborg Nordmann. Sie haben mit zahlreichen, auf allgemein interessierte Leserschaften abgestellte Brief- und Texteditionen ein wesentlich komplexeres Bild von der Person und dem Werk Arendts geschaffen als dies vor 1990 noch der Fall war. Erinnert sei hier auch an den früh verstorbenen Essayisten Eike Geisel, der für zahlreiche Übersetzungen bis dahin nicht auf Deutsch zugänglicher Texte sorgte.

In den USA sind die von Jerome Kohn, einem Schüler und Freund Arendts, publizierten Sammelbände (zuletzt Arendt 2018a) neben ihren bekannten Monografien häufig der Ausgangspunkt für die Rezeption. Gleiches gilt für Südamerika, den Staaten des früheren Ostblocks, aber auch im asiatischen Raum, in denen immer mehr Übersetzungen in die jeweilige Landessprache erscheinen. Mit „hannaharendt.net“ und den „Hannah Arendt Studies“ sind viel genutzte Online-Plattformen entstanden, die neben Analysen auch ständig neue Quellen zu Arendt im Internet publizieren.

Die jüngste und jede weitere Beschäftigung mit Arendt entscheidend beeinflussende Nachricht lautet, dass zwei Bände einer kritischen Ausgabe ihrer Werke erschienen sind. Die alle Schriften, Manuskripte, Varianten, Fragmente und Notizen in Buch- und digitaler Form umfassende Edition wird durch die Deutsche Forschungsgemeinschaft (DFG) langfristig gefördert. Die von der seit vielen Jahren sich mit Hannah Arendt und deren Themen auseinandersetzende Literaturwissenschaftlerin Barbara Hahn maßgeblich initiierte Ausgabe wird damit endlich zuverlässige Textgrundlagen schaffen können und damit hoffentlich die noch immer die Rezeption massiv negativ beeinflussende Trennung in die auf Englisch und in die auf Deutsch schreibende Arendt beenden. Auf dieses Thema werde ich noch ausführlicher eingehen.

Aus dem Gesagten wird deutlich, dass bei der Auswahl der Literatur aus den letzten fünf Jahren Schwerpunkte gesetzt werden mussten. Sie ergeben sich aus den derzeitigen Forschungsinteressen und aus der Tatsache, dass die lange erwartete kritische Ausgabe der Schriften Arendts mit den ersten Bänden aufwarten kann.

Zunächst werden also neue Ausgaben von Arendts Texten behandelt (2), dann folgen Einführungen und Überblickswerke (3) dann ihre Beschäftigung mit der Antike (4), der Literatur (5) und schließlich dem Recht (6).

\footnotetext{
2 Nicht näher eingegangen werden kann auf ein Buch wie das von Elisabeth Gallas, A Mortuary of Books. The Rescue of Jewish Culture after the Holocaust, New York 2019, in dem erstmals gänzlich aus Archiven geschöpft die Geschichte der ,Jewish Cultural Reconstruction“(JCR)-Organisation geboten wird. Arendt spielte in ihr in verschiedenen Funktionen eine entscheidende Rolle. Diese Funktionen hatten einen maßgeblichen Einfluss auf ihr erstes Hauptwerk: die Origins.
} 


\section{Neue Schriften von Hannah Arendt}

Die wichtigste Neuerscheinung stellt der in der PVS bereits ausführlich vorgestellte Band Modern Challenge to Tradition (Arendt 2018b) dar, der zugleich der erste Band der kritischen Ausgabe ist. ${ }^{3}$ Die zwischen dem Juni 1952 und September 1954 entstandenen Texte liegen zeitlich zwischen der Erstausgabe von Origins of Totalitarianism bzw. Burden of our Time (1951) und der von Arendt selbst übersetzten und dem vermeintlichen Original gegenüber wesentlich veränderten deutschen Ausgabe Elemente und Ursprünge totaler Herrschaft (1955).

Wie Ahlrich Meyer überzeugend herausgearbeitet hat, liegt die Bedeutung des Bandes nicht an Arendts Marx-Analysen (Meyer 2019), die sich in einem relativ enggesteckten Rahmen bewegen. Bedeutsam ist vielmehr die Denkbewegung, die sich in dem Band dokumentiert. Ausgehend von der bekannten TotalitarismusAnalyse, wie sie in den Origins entwickelt wurde, sucht Arendt in den in Modern Challenge to Tradition gesammelten Texten die Voraussetzungen für ein ehrgeiziges, ihre weiteren systematischen Interessen bestimmendes Vorhaben zu schaffen. Dass dieses Vorhaben in verdichteter Form unter anderem in einem Brief an Martin Heidegger vom 8. Mai 1954 formuliert wurde, stimmt auf den ersten Blick. ${ }^{4}$ Darin hatte Arendt drei, voneinander unabhängige Ideen entwickelt, die sie künftighin verfolgen wolle. Zunächst eine „Analyse der Staatsformen“, die mit Montesquieu einsetzt. Sodann eine bei Marx und Hobbes gleichermaßen ansetzende Theorie, die die Kategorientrias Arbeiten, Herstellen, Handeln im Zentrum haben sollte. Und schließlich eine aus der Deutung des sogenannten platonischen „Höhlengleichnisses“, in Anlehnung an Heideggers Auslegung, hervorgehende Prüfung dessen, was Arendt ,politische Philosophie“ nannte.

Diese dreifache Aufgabe sollte in drei Büchern erfolgen: einer Einführung in die Politik, die sowohl in der deutschen wie der amerikanischen Fassung sehr fragmentarisch blieb; die Ansätze zu einem Buch über Marx, das weit über Marx' Theorien hinaus wollte, sind in Modern Challenge dokumentiert, während das Hobbes-Buch leider eine bloße Idee blieb, die auf Waldemar Gurian zurïckging; die Vorlesungen im Rahmen der Walgreen Lectures an der University of Chicago führten letztlich zu Human Condition bzw. Vita activa, allerdings mit einer scharfen Kritik an Heideggers „Höhlengleichnis“-Interpretation.

Wesentlich ist hier nicht ihre Ignoranz des Planes als vielmehr das gegenüber Heidegger Verschwiegene. Arendt befand sich nämlich auf dem Weg zu einer neuen politischen Wissenschaft, deren Neuartigkeit letztlich in der Restituierung eines aristotelischen Modells stand, in dem eine anthropologisch fundierte Ethik (Human Condition/Vita activa) in eins mit einer auf die Idee der Republik (On Revolution/ Über die Revolution) zielenden Staatstheorie stand, die in Life of the Mind handlungstheoretisch hätte ausformuliert werden sollen. Nichts von alldem ist auch nur im Entferntesten ,heideggerianisch“.

\footnotetext{
3 Siehe Winfried Thaa in der Politischen Vierteljahresschrift (2019, Jg. 60, Heft 3), S. 623-628.

4 Hannah Arendt und Martin Heidegger, Briefe 1925 bis 1975 und andere Zeugnisse. A. d. Nachlass hg. v. Ursula Ludz.(1998, S. 146)
} 
Wie das informative Nachwort des ausführlich kommentierten Bandes belegt, war die Edition alles andere als eine einfache Angelegenheit: Das Nachlassmaterial wurde mehrfach umsortiert und es war nahezu unmöglich, Arendts ursprüngliche thematisch-chronologische Ordnung zu rekonstruieren. Umso erstaunlicher ist die vorliegende Geschlossenheit von Modern Challenge. Wer den in jeder Hinsicht reichen Band konsultiert, sollte allerdings auch die kritischen Überlegungen im Hinterkopf haben, die Ursula Ludz (Ludz 2020) im Politischen Jahrbuch anstellte. Nach Ludz' Analyse folgen die Anordnung und die Analyse einer von den Herausgebern vorgegebenen Konstruktion, nämlich dem nicht geschriebenen und dann vielfach variierten Buch zu Marx als „Modern Challenge“.

Der zweite Band innerhalb der kritischen Ausgabe bietet die Texte, die erstmals 1948 in dem Sammelband Sechs Essays erschienen (Arendt 2019b) und die von ihr selbst in veränderte/erweiterter Zusammenstellung unter dem Titel Die verborgene Tradition auf Anregung des Schriftstellers Uwe Johnson neu herausgegeben wurden. Der Band bietet wie Modern Challenge alle Originaltexte in deutscher und englischer Sprache sowie ausführliche Erläuterungen und Kommentare. Leider wurde in dem Band der für Arendts Sicht des Staatsbildungsprozesses Israels äußerst wichtige Text Zionism Reconsidered von 1945 in einer von Arendt nicht autorisierten, passagenweise mangelhaften Übersetzung ins Deutsche abgedruckt.

Mit der Entscheidung die Sechs Essays bzw. Die verborgene Tradition an den Anfang der Neuausgabe bereits veröffentlichter Schriften zu setzen, ist eine doppelte Herausforderung für künftige Interpreten gegeben. Zum einen werden sie, die üblicherweise die Totalitarismus-Theoretikerin allmählich zur Philosophin werden lassen, zugeben müssen, dass Arendt tatsächlich mehrere, davon unabhängige Themen gleichgewichtig nebeneinanderstellt. Zum anderen wird in der Folge neu zu erklären sein, inwieweit Arendt überhaupt als Systematikerin im herkömmlichen Sinne verstanden werden kann. Denn Letzteres scheint noch die Ambition vieler Interpreten zu sein. Life of the Mind als Zielpunkt kann vorerst sowieso ausgeklammert werden, da schon ein erster Blick in den Nachlass zeigt, dass Mary McCarthy an den editorischen Aufgaben scheiterte und die vorliegende Ausgabe nicht als Arendts Text zu werten ist.

Unter den in jüngerer Zeit vorgelegten Editionen von Texten und Briefen Hannah Arendts sind die Korrespondenzen mit fünf engen Freundinnen und der Textband Wir Juden sicherlich die wichtigsten (Arendt 2017 bzw. Arendt 2019a). Beide Bände verstehen sich als Leseausgaben. Die von Ludz und Nordmann herausgegebenen Korrespondenzen mit Charlotte Beradt, Rose Feitelson, Hilde Fränkel, Anne Weil und Helen Wolff umfassen die Zeit von den frühen 1940er-Jahren bis zu Arendts Tod 1975. Die Einführungen und Kommentierungen vermitteln einen guten Eindruck von Arendts Lebenslagen und Schreibsituationen. Für die Arendt-Forschung sind die Bände insofern von besonderem Belang, als sie eindrücklich belegen, wie sehr in ihrem Falle Persönliches und Denken miteinander korrelieren. Über die Bedeutung von „Freundschaft“" in Arendts Denken ist sehr viel geschrieben worden, in dem Band mit ihren Freundinnen lässt sich das nunmehr genauer nachvollziehen, wie sich in konkreten Situationen die konzeptionellen Überlegungen bewähren - oder eben nicht. 
Dass das Briefwerk, insbesondere der Austausch mit Karl Jaspers, Heidegger und ihrem Ehemann Heinrich Blücher ${ }^{5}$ werden immer wieder herangezogen, Teil des vermeintlich eigentlichen Werkes ist, stand sowieso nie in Frage. Die Frage war vielmehr, welche Idee(n) von Freundschaft sich aus den persönlichen Beziehungen und den dort praktizierten Diskretionsformen herauspräparieren lassen. Inwieweit Arendts Vorstellung von ,,amor mundi““ auf ,philia“-Konzeptionen zurückgeht, ließe sich mit dem „Freundinnen“-Band nunmehr weitaus besser analysieren.

Dass die Herausgeberinnen, darin leider einer gängigen Praxis folgend, in Briefe eingegriffen und andere nicht abgedruckt haben, ist unverständlich. Wurde schon in früheren Bänden, insbesondere im Falle Arendt-Blücher, eine schlingernde, nie konsistente Politik von Abdruck und Auslassung verfolgt, so findet das hier eine leider unklar bleibende Fortsetzung. Damit ist in keiner Weise unterstellt, dass wichtiges Material unterschlagen wird. Gerade im Falle Arendt-Blücher kann sich jeder im Archiv vergewissern, dass das nicht der Fall ist. Durch die zwar im Hinblick auf noch lebende Personen verständliche Editionspolitik - Arendt und ihre BriefpartnerInnen schonten andere nicht - werden aber, auch das könnte am Briefwechsel mit Blücher exemplarisch gezeigt werden, wichtige Hinweise eliminiert.

Anders gelagert ist der Fall des Briefwechsels zwischen Dolf Sternberger und Arendt (Arendt und Sternberger 2019), der jetzt erstmalig von Udo Bermbach herausgegeben wurde. Arendt und Sternberger kannten sich aus der gemeinsamen Zeit in Heidelberg, also seit der zweiten Hälfte der 1920er-Jahre. Die enge, auch intellektuelle Freundschaft endete mit der „Machtergreifung“ der Nationalsozialisten. Wobei die Frage ist, warum sich aus der Zeit vor 1945 keinerlei Dokumente finden lassen. ${ }^{6}$ Sternberger war bei Paul Tillich in Frankfurt mit einer Heidegger-kritischen Dissertation promoviert worden, die 1934 unter dem Titel Der verstandene Tod erschien. Man könnte den Briefwechsel in seinen systematisch interessanten Teilen als fortwährende Auseinandersetzung um den Status von Heideggers Denken lesen. Hier prallen, bei aller persönlicher Sympathie, Gegensätze aufeinander: Arendt wird stets Heideggers Versuch einer vollständigen, radikalen Ersetzung der klassischen „Philosophie“ durch das, was er das „Denken“ nennt, verteidigen. Und das schließt ausdrücklich die nicht minder radikale Kritik mit ein, die sie selbst an dem emphatischen Seins-Denken übt. Dass die Edition, die Bermbach mit einer informativen Einleitung versehen hat, selbst den schlichtesten Kriterien einer Leseausgabe nicht gerecht wird, wurde bereits vielfach angemerkt. ${ }^{7}$ Hier sei nur ergänzt, dass ein wichtiger Brief fehlt, die Transkription alles anders als sauber ist, ein anderer Brief zweifach abgedruckt wurde und sich auch unbegründete Auslassungen finden - und

\footnotetext{
$5 \mathrm{Zu}$ Blücher sei auf folgende Pionierarbeit verwiesen: Heinrich Blücher, Versuche über den Nationalsozialismus. Hg. von Ringo Rösener. Mit einem Nachwort von Ringo Rösener und Eyck-Marcus Wendt, Göttingen 2020.

6 Wie Recherchen im Deutschen Literaturarchiv belegen, findet sich Arendts Name nicht vor deren Emigration in den Nachlasspapieren Sternbergers. Umgekehrt taucht Sternberger nicht in den nur wenigen erhaltenen Dokumenten Arendts aus dieser Zeit auf. Ich danke Georg Hartmann vom DLA Marbach für die Auskunft.

7 Siehe dazu Jens Hacke (2020), S. 123-127. Siehe zudem die Kritiken, die Gustav in der „Süddeutschen Zeitung“, Alexander Gallus in der „FAZ“ und der Autor in der „FAS“ veröffentlicht haben.
} 
ausgerechnet der so von Arendt und Sternberger geschätzte Aristoteles zum Autor von Platons Politeia mutiert.

Unter dem Titel Wir Juden (2019a) haben Knott und Ludz eine Auswahl von Texten aus den Jahren von 1932-1966 versammelt, die die beiden Herausgeberinnen den sogenannten ,jüdischen Schriften“ zuschlagen. Eine ähnlich gelagerte Idee hatten Kohn und Ron H. Feldman 2008 mit ihrem Band der Jewish Writings, der auch Archivmaterial heranzog und einen Text präsentierte, dessen Autorin nicht Arendt ist (2007). ${ }^{8}$ Wir Juden lässt unabhängig von Fragen der Auswahlkriterien den Zusammenhang von Arendts Verhältnis zu ihrem Jüdisch-Sein, dem Judentum und den Judentümern, dem jüdischen Denken, dem Holocaust, Palästina/Israel und zur israelischen Politik auch dank der beigegebenen Kommentare besser verstehen. Das heißt konkret: Die geschichtliche Entwicklung zieht Arendt in die Notwendigkeit hinein, ihre Identität präziser bestimmen zu müssen. Mit Identität ist aber nicht das bloße Ich gemeint, sondern die Korrelation zwischen der jüdischen Frau und den Judentümern, Juden und Nichtjuden, den geschichtlich gewachsenen Kategorien, um diese Verhältnisse bestimmen zu können und deren mögliche Revision. Arendts Denken durchläuft diesbezüglich in den Jahrzehnten verschiedene Stadien, die in Wir Juden abgebildet werden. Wohl des Umfanges wegen wurde ein mit „Antisemitismus“ überschriebenes Manuskript nicht abgedruckt, ${ }^{9}$ das gleichwohl in englischer, wie französischer Übersetzung vorliegt. Die wohl aus dem Jahr 1938, auf Vorlesungen aus dem Vorjahr beruhende Studie stellt die Keimzelle der Origins of Totalitarianism dar.

Der von Jerome Kohn herausgegebene und eingeleitete Sammelband Thinking Without a Banister (Arendt 2018a) ist nicht unproblematisch. Die zwischen 1953 und 1975 entstandenen Texte sind zum größten Teil publizierte, in Zeitschriften oder Sammelbänden leicht zugängliche Arbeiten. Einige Texte wurden bereits mehrfach abgedruckt. Zudem hat Kohn unveröffentlichte Vorträge und Briefe bzw. kurze Briefwechsel eingestreut. Anders als frühere Zusammenstellungen lässt sich in dem neuen Band keine Kohärenz ausmachen, es sei denn, man zieht sich auf den Standpunkt zurück: Alles von Arendt sei interessant und je mehr vorliege, desto klarer wäre ihre Denkentwicklung. Ein anderer möglicher Standpunkt ist zu sagen, dass Arendt auch noch in ihren scheinbar marginalsten Arbeiten ihre Grundgedanken ausprobierte und variierte. Beides stimmt natürlich und darauf seit Jahrzehnten hingewiesen zu haben ist Kohns uneingeschränkter Verdienst. Thinking Without a Banister ist insofern als Ergänzung zu den früheren Sammelbänden zu bewerten.

Zugleich wirft der Band ein Problem auf, das nur durch die entstehende kritische Ausgabe gelöst werden kann: ${ }^{10}$ Es gibt inzwischen ein kaum mehr zu überschauendes Sammelsurium an Auswahlbänden mit Schriften Arendts. Wiederabdrucke von

\footnotetext{
${ }^{8}$ Hier handelt es sich um einen Text, der 1936 in Die neue Weltbühne unter dem Titel „Prozeß Gustloff“" (S. 1602-1604) publiziert wurde. Das verwendete Pseudonym „Helveticus“ wurde abwechselnd zwei männlichen Autoren zugewiesen, wie der nur in kleinen Teilen erhaltene Nachlass der Weltbühne belegt.

9 Das entsprechende, wohl 1937/38 geschriebene Manuskript ist 159 Seiten lang.

${ }^{10}$ Der Verfasser weist aus Transparenzgründen darauf hin, dass er eine „Studienausgabe“ im Münchner Piper Verlag herausgibt, die in einer ersten Tranche die Monografien bzw. die von Arendt selbst herausgegebenen Sammelbände bietet. Der Herausgeber hat die Texte durchgesehen und KollegInnen gebeten, umfängliche „Nachworte“ beizusteuern.
} 
Texten, deren „Weg“ nicht nachzuvollziehen ist, Zusammenfassungen von Aufsätzen, Vorträgen, Gelegenheitsarbeiten, die eine kontinuierliche Arbeit an „Problemen“ suggerieren, die Arendt so nicht leistete. Gerade das wird in der Regel nicht offengelegt, das heißt: Dass hinter den Textkompilationen eine Vorannahme steht, verschwindet hinter der oder den Thesen, die von HerausgeberInnen in Einleitungen oder Nachworten vertreten werden. Ein so simples historistisches Prinzip wie die Chronologie wird zugunsten einer scheinsystematischen Auflösung von Kontexten gar nicht erst in Erwägung gezogen. Gerne beruft man sich dabei auf Arendt selbst, die vermeintlich das Experimentelle dem Systematischen vorgezogen habe. Deshalb auch die ständigen Hinweise auf ein „Denken ohne Geländer“ oder ,Ich will verstehen“. Dass Arendt hier Selbstverständlichkeiten wiedergibt, einmal das Grundpostulat philosophischen Denkens seit Sokrates und dann die Kernaussage der traditionellen Hermeneutik, wird nicht nur ausgeblendet, sondern als konstitutiver Teil einer spezifischen Originalität behauptet. Die politische Theoretikerin wird so ,populär“ dargeboten und damit letztlich ihr Denken verzeichnet.

\section{Companions, Einführungen und Gesamtdarstellungen}

Bevor auf einzelne Studien eingegangen wird, sollen ein paar grundsätzliche Bemerkungen zum Stand der Sekundärliteratur gemacht werden.

Es gibt eine für die Erforschung von Arendts Werk erstaunliche Zweiteilung: ForscherInnen, die das deutschsprachige Werk Arendts in ihre Überlegungen einbeziehen und solche, die es nicht tun. Nun mag zugegeben werden, dass nahezu alle auf Deutsch verfassten Texte auf Englisch, der ,,anderen“ Sprache Arendts - auf Französisch hat sie nur zwei kleinere Artikel in den 1930er-Jahren geschrieben ${ }^{11}$ zur Verfügung stehen. Doch so einfach verhält sich die Sache bei Weitem nicht. Während es seit gut 20 Jahren in der deutschsprachigen Forschung Reflexionen zur Frage nach dem Status des Deutschen und Englischen im Werk Arendts gibt, fehlt dies in nichtdeutschsprachigen Publikationen vollends. Das ist umso überraschender, als Arendt immer wieder seit ihrer Emigration das Verhältnis von Sprache und Denken thematisierte und damit zu einem wichtigen Gegenstand ihres Werkes machte. Alle ihre zentralen Texte, insbesondere die großen Monografien, übertrug Arendt selbst ins Deutsche bzw. sorgte für die Veröffentlichung einer deutschen Version. Aber das ist eine bloße Feststellung, denn darunter liegt ein komplexes Wechselverhältnis zwischen den beiden Sprachen und den Inhalten. Man schaue sich nur die verschiedenen Fassungen der Origins bzw. Elemente an: Hier lässt sich mit der bloßen Aufzählung von Veränderungen allenfalls ein formales Argument bilden. Die Umschreibungen sind vielmehr wesentlich für Arendts Methodik - die man gewiss

\footnotetext{
11 Das heißt nicht, dass Arendt nicht aufmerksam den französischen „Markt“ bediente. So ist ihr ursprünglich 1945/46 auf Deutsch verfasster Aufsatz „Was ist Existenzphilosophie?“, der zunächst auf Englisch in der Partisan Review? 1946 erschien, auf Französisch 1947 - in der Übersetzung ihrer Freundin Catherine Mendelsohn - publiziert worden. Und das in einer von der ein Jahr später vorgelegten deutschen Version abweichenden Fassung.
} 
in verschiedener Weise charakterisieren kann. Aber man darf über die genannten Phänomene nicht schweigen, vielmehr muss man sie zu verstehen suchen.

Das Gesagte mag auf den ersten Blick nationalchauvinistisch klingen. Natürlich ist mit der gemachten Feststellung in keiner Weise etwas über die Qualität der nichtdeutschsprachigen Forschung gesagt. Man könnte stattdessen dialektisch behaupten, dass aus der Ignoranz der Sprach- und Übersetzungsproblematik ein Gewinn erzielt wird: nämlich die Konzentration auf die auf Englisch vorliegenden Texte. Doch diese Leerstelle, die in anderen Fällen mal als verstörend, mal als Ignoranz ausgelegt werden kann, ist bei Arendt in jedem Falle ein interpretatorisches Problem. The Human Condition ist ein anderes Buch als Vita activa - ein Umstand, der, hier lediglich exemplarisch herausgegriffen, durch die kritische Edition noch viel deutlicher werden wird. Der Sprachwechsel ist verbunden mit einem völlig anderen terminologischen Feld, in dem gänzlich andere Bedeutungen transportiert werden. Und gerade in dem Maße, wie Arendt (rück-)übersetzt, wird deutlich, wie sehr sie dem deutschen terminologischen Feld verbunden bleibt, wenn sie auf Englisch schreibt. Den Wechsel der Sprache nicht zu reflektieren und dabei auch die vorgenommenen, teilweise massiven Veränderungen gegenüber den englischen „Originalen“ nicht in die Analyse mit einzubeziehen, heißt letztlich nur die sprichwörtlich „halbe“ Arendt in den Blick zu nehmen.

Es ist weiterhin erstaunlich, dass es noch immer keine Gesamtdarstellung von Arendts Denken gibt, egal in welcher Sprache, die systematisch ihren Nachlass einbezieht. Überhaupt ist festzustellen, dass nicht nur der Nachlass ausschließlich dann in den Blick gerät, wenn für die jeweilige Fragestellungen „Interessantes“ oder „Neues“ gesucht wird. Auch hier wird die kritische Ausgabe für künftige Arbeiten eine hermeneutisch sehr viel höhere Hürde aufbauen. Denn neben dem häufig unhistorischen Zugriff auf den Nachlass überrascht die fehlende Quellenkritik. Briefe, Vorträge, Veröffentlichtes, Nichtveröffentlichtes werden in der Regel gleichrangig als „Äußerungen“ verarbeitet. Hier fällt die neuere Forschung nahezu ohne Ausnahme unisono gegenüber älteren Arbeiten zurück, wie überhaupt zu beobachten ist, dass sich Historiker und damit auch die Methoden der Geschichtsschreibung aus der Arendt-Forschung zurückziehen. ${ }^{12}$

Das ist eine Beobachtung, keine Wertung. Neuere und unbedingt lesenswerte Gesamtdarstellungen sind von PhilosophInnen und PolitikwissenschaftlerInnen gleichermaßen geschrieben. Die Berliner Habilitationsschrift von Stefania Maffeis (Maffeis 2018), zusammen mit der umgearbeiteten, auch stilistisch brillanten Dissertation von Maike Weißpflug (Weißpflug 2019), und den grundlegenden Einführungen von Hans Jörg Sigwart (Sigwart 2016) sowie Grit Straßenberger (2015) und schließlich der knappen und zugleich dichtgewobenen Darstellung von Jana V. Schmidt (2018) bieten auf je unterschiedliche Weise Zugänge zu Arendts Gesamtwerk.

Straßenberger beginnt dort, wo Arendts Denken politiktheoretisch interessant wird: bei deren Totalitarismusanalysen. Hier, wie in den anderen zentralen Fragen, die an eine ,politische Theoretikerin“ zu stellen sind, geht es Straßenberger immer um die innere Kohärenz von Arendts Überlegungen. Dabei wird sie stets

12 Ich denke hier etwa an die Arbeiten von Claudia Althaus, Claudia Christophersen, Wolfgang Heuer, Iris Pilling oder Annette Vowinckel im deutschsprachigen Raum. 
„stark“ gemacht und zugleich wird ihr immenses Werk auf Grundzüge reduziert. Politik, Handlungstheorie, Aristotelismus - damit sind die Felder abgesteckt, die Straßenberger präzise durchgeht, auch um die zahlreichen Verschiebungen innerhalb von Arendts Denken in den Griff zu bekommen. Dass die Autorin gegenüber ihrer Heldin skeptisch bleibt, hat genau dargelegte Gründe. Sie hält sich an das, was grundsätzlich gilt: Wer Arendt aktualisieren möchte, der muss immer angeben, welche Arendt gemeint ist. Eine irgendwie geartete ,ganze“ Arendt ist weder zu rekonstruieren noch wäre eine solche Rekonstruktion in die Gegenwart zu retten.

Alle weiteren Einführungen blicken quasi von der Seite auf Arendt und damit in anderer Weise als der „Gründer“ dieses Genres: Hauke Brunkhorst, der sich an ihren prominenten Schriften orientierte. ${ }^{13}$ Unsere InterpretInnen beginnen von einem nur scheinbar mehr oder weniger randständigen Aspekt. Damit wird zunächst der Tatsache Rechnung getragen, dass Arendts ,Werk“ eines der beständigen Verschiebungen und Veränderungen ist. Es gibt keinen zentralen Begriff, beispielsweise Denken, Gewalt, Handeln, Macht, Revolution, der unverändert geblieben wäre. Gesamtdarstellungen müssen also entweder immense Dynamiken innerhalb von Arendts Schriften darstellen oder aber auf ganz basale, siehe die Auswahl von Zentralbegriffen, Annahmen der politischen Theoretikerin vertrauen. In dieser scheinbaren Zwangslage erweisen sich die Arendt-KennerInnen als äußerst flexibel. Der Start vom vermeintlichen Rand in die Zentren erfolgt gänzlich unabhängig von Arendts Methoden und gerade dadurch werden neue Zugänge eröffnet: In Maffeis umfangreichen Rekonstruktionen von Arendts Denkwegen ist es die hier erstmals mitgelieferte Rezeptionsgeschichte, die, von der Autorin mit entschiedenen Urteilen versehen, eine wesentliche Leerstelle in der Forschung füllt und auf diese Weise die Texte in ein neues Licht stellt. Damit wird nicht nur das noch immer kursierende Gerücht einer „späten“ Entdeckung Arendts widerlegt, vielmehr wird gezeigt, wie früh sie immer auch für ihre Argumentationen in Grundlegungsfragen der politischen Wissenschaft ernst genommen wurde. Das ist das vielleicht erstaunlichste Ergebnis von Maffeis Lektüren.

Nicht minder wichtig ist Weißpflugs Studie, deren Untertitel „Die Kunst, politisch zu denken“" prägnant ein Element hervorhebt, das noch immer unterbelichtet ist in der Arendt-Forschung: nämlich ihre dynamischen Denkprozesse - und Deutungsänderungen als etwas Ästhetisches zu begreifen - ohne in die Falle einer Ästhetisierung zu laufen. Weißpflug wiederum achtet sehr genau darauf, dass die erwähnte „Kunst“ immer im Dienste des Politischen steht, also nicht die in der politischen Philosophie immer wieder anzutreffende Position verteidigt, dass das angeblich vernachlässigte Ästhetische an die Stelle eines bloß behaupteten genuin Politischen oder des Rechts treten solle. ${ }^{14}$ Vielmehr orientiert sich Weißpflug mit Arendt an verschiedenen Lektürepraxen, die etablierte Genregrenzen durchbrechen. Dabei geht die Interpretin so weit, dass sie dem politischen Denken Arendts nicht nur die offensichtlichen literarischen Elemente und Erzählstrategien zuschreibt, sondern dieses selbst als Narration

\footnotetext{
${ }^{13}$ Die Einführung des Flensburger Politikwissenschaftlers: Hannah Arendt. Eine Einführung, München 1999, ist noch heute unbedingt lesenswert.

${ }^{14}$ Hierfür stehen cum grano salis die Arbeiten von Christoph Menke, Juliane Rebentisch und deren SchülerInnen.
} 
auftritt. Das von Weißpflug gewählte Verfahren ist auch deshalb aufschlussreich, weil sie die blinden Flecken bei Arendt im Blick hat. Dabei sticht vor allem die Analyse des Aufsatzes über die sogenannten ,Rasseunruhen“ in „Little Rock“ heraus, die zeigen kann, wie sehr Arendt hier eine Kategorie außer Acht ließ, deren Bedeutung in ihrem Werk unübersehbar ist: Kommunikation. Arendt versagte, mit Weißpflug formuliert, an mangelnder ,Responsivität““. ${ }^{15}$

An dieser Stelle wird Jana V. Schmidts Büchlein wichtig. Sie beendet den analytischen Teil mit dem bekannten Zitat: „Gibt man die Urteilskraft aus der Hand, wird in der Tat alles Schwindel.“ Genau das war die Ausgangslage für Weißpflug, die bei Arendt eine Weitung des Urteilsbegriffs - hier wörtlich zu nehmen als einer erkenntnisleitenden Kraft -, feststellte, die jenseits der, ,richtig/falsch“-Struktur liegt und auf das (kommunikative) Handeln verweist. Schmidt wiederum sieht darin das Ergebnis einer von ihr knapp, präzise und ganz und gar eigenständig wiedergegebenen Denkentwicklung. Einer Entwicklung, die im sozialen Zwischen begann und mit einer dezidiert antiprozessuralen Position endete: einer Art reflektiertem Situationismus. Was könnte damit gemeint sein? Anders als etwa in Heideggers Fundamentalontologie kennt Arendt den für das Politische und das Soziale konstitutiven Moment der Spontaneität. Etwa dann, wenn sie in Vita activa den „Erscheinungsraum“, also dort, wo Politisches und Soziales in der menschlichen Begegnung kreuzen, als Wechselspiel von Zufall und Absicht charakterisiert.

Damit wären wir bei Sigwarts Einführung, die zugleich weitaus mehr ist. Er beginnt mit einem erstaunlichen Zitat, das in den zahlreichen Transkriptionen des berühmten Fernsehinterviews mit Günter Gaus aus dem Jahr 1964 fehlt. Die Formulierung Arendts, „dass man sich nicht selber in die Karten schauen soll“, ließ man einfach verschwinden. Dabei ,,verrät“ Arendt mit der Redewendung ihr Programm. Sigwart erkennt völlig zu Recht in der bislang unterdrückten Passage einen erkenntnistheoretisch und hermeneutisch wichtigen Tatbestand: die Unmöglichkeit der völligen Transparenz des Selbstverhältnisses und des von einem Subjekt Gedachten. Der Interpret hingegen muss, um im Bild zu bleiben, in die Karten derer schauen, die sie selbst nicht sehen kann - und Sigwart tut dies mit einem vermeintlich eingeschränkten Erkenntnisinteresse. Er möchte die angesprochene Dynamik, von ihm im Bild des ,wandering“ gefasst, von Arendts Denken offenlegen, wie es sich im von ihr geschaffenen Zwischenraum von Politik und Philosophie bewegt. Und jener Zwischenraum wird als das Kreuzungsfeld von Vita activa und Vita contemplativa vermessen. In diesen Zwischenraum packt Arendt dann die Welt und deren Gehalt, der sich sowohl in seinen facettenreichen Zusammenhängen als auch den zahlreichen Perspektiven, mit denen man auf die Zusammenhänge blickt, manifestiert. Dieser Zwischenraum ist der, und damit wird ein weiterer Aspekt dieses Begriffs deutlich, „Erscheinungsraum“ - in diesem Falle das, was von Menschen handelnd gemacht und sodann darüber geurteilt wird. Mit diesem Konstrukt strebt Sigwart eine Gesamtschau auf Arendts Werk an.

\footnotetext{
15 Zentral für jedwede Beschäftigung mit Arendts „Little Rock“-Aufsatz ist: Kathyrn T. Gines, Hannah Arendt and the Negro Question, Bloomington (2014). Gaines' „close-reading“ kann als beispielhaft für eine kritisch-hermeneutische Auseinandersetzung mit Arendt gelten.
} 
Dass Hannah Arendt kritisch auf die Soziologie ihrer Zeit blickte, ist bekannt. Eine systematische Rekonstruktion ihrer Kritik an soziologischen Kategorien und Methoden fehlte bislang jedoch. Doch nicht nur dieses Desiderat will nun eine präzise aufeinander abgestimmte Sammlung von Aufsätzen auflösen. Die AutorInnen loten aus, wie aus Arendts Kritik an der Soziologie ein Zugewinn für das Fach werden könnte. Dies ist das erklärte Ziel des Anthem Companion to Hannah Arendt (Baehr und Walsh 2016). Die mit der eigenständigen „Introduction“ der beiden Herausgeber zusammen zehn Beiträge bilden eine konzise Rekonstruktion von Arendts Umgang mit soziologischen Theorien, verfolgen diese von den Origins bis hin zu Life of the Mind, um sich anschließend Fragen von „Thinking“, „Genocide“, der Beziehung von „Power and Violence“ und schließlich dem „Totalitarian Leadersphip“ zu widmen. Baehr, Walsh und ihre MitstreiterInnen belegen, dass Arendts oftmals zeitbedingte Ablehnungen gegenüber anderen Disziplinen - neben der Soziologie wäre hier vor allem die Psychologie zu nennen - sich durch konsequente Dekonstruktionen durchaus in Anregungen für deren Problemstellungen transformieren lassen.

\section{Arendt und die Antike}

Ein weiterer eklatanter Mangel innerhalb der Arendt-Literatur ist die Beschäftigung mit antiken Autoren. Seitdem der 2019 verstorbene Jacques Taminaux Arendt und Heidegger 1992 im Hinblick auf die Antike untersuchte und Thomas Gutschker zehn Jahre später Arendts Beziehung zu Aristoteles analysierte, ${ }^{16}$ war es vor allem Agamben, der, im Rahmen seines nunmehr abgeschlossenen Homo sacer-Projekts, an die Tiefendimension von Arendts Platon- und Aristoteles-Lektüren erinnerte. In der für ihn typischen radikalen und damit auch hochproblematischen Einseitigkeit, machte er die bei Arendt im Anschluss an Aristoteles' Nikomachischer Ethik prominent vorkommende Unterscheidung von bios und zoé zum Ausgangspunkt seiner Überlegungen. ${ }^{17}$

Damit mag ein wichtiger Aspekt von Arendts Lektüren antiker Autoren erfasst sein, mehr aber auch nicht. Es fehlt weiterhin ein Versuch, der ihre Auseinandersetzungen mit Platon und Platons Sokrates, Aristoteles, aber auch Sophokles, Euripides und Aischylos in einen Zusammenhang bringt. Das hat zahlreiche Gründe: Arendts Verwurzelung im antiken Denken wird zwar immer betont, doch selten finden sich die Kompetenzen oder das Interesse, dem genauer nachzugehen. Die Voraussetzungen, um in diesem für das Verständnis von Arendts Denken zentralen Feld Fortschritte erzielen zu können, sind nicht nur durch die Nachlasserschließung gegeben, sondern auch durch die voranschreitende Digitalisierung von Arendts Bibliothek, die am Bard College realisiert wird. Dabei wird deutlich, wie sehr sie sich

\footnotetext{
16 Siehe dazu auch Jacques Taminaux (1992) und Thomas Gutschker (2002).

17 Giorgio Agamben (2002), S. 11. Seitdem gibt es Regalmeter an Sekundärliteratur, die die von Altphilologen und Aristoteles-Spezialisten zurückgewiesene Unterscheidung Arendts und Agambens, weil zu streng und nicht systematisierbar, für freischwebende Spekulationen adaptiert haben. Eine Übersicht bietet: Eva Geulen (2016).
} 
in der Antike bewegte, die die selbstverständliche Basis für ihre Überlegungen bildete. Dass im antiken Athen die Philosophie ihre Möglichkeiten genuin politisch zu denken verabsäumte, eine Überlegung, die das gesamte Werk seit der Flucht in die USA durchzieht, lässt sich sogar als Grundeinsicht Arendts bezeichnen. Die Rolle der griechischen Literatur für die nach und nach entwickelte Handlungstheorie lässt sich vor diesem Hintergrund gar nicht hoch genug einschätzen.

Man betrachte etwa das Ende von On Revolution/Über die Revolution. Die Studie kulminiert mit einem Zitat aus Sophokles' Ödipus auf Kolonos (Verse 1225-1228), das Arendt im Original sowie in der vermeintlichen Übersetzung von Karl Reinhardt wiedergibt. Damit nicht genug: Die letzte Zeile enthält wiederum ein Zitat, wiederum auf Altgriechisch, dieses Mal von ihr eigenwillig übersetzt. ${ }^{18}$ Es ist also klar, dass nicht Geschichte, Philosophie oder eine revolutionstheoretische, also letztlich republikanische Idee das Buch abschließt, sondern ein höchst enigmatischer Vers aus der letzten Tragödie des Sophokles, der einen Theseus sprechen lässt, der eine Art ungeschichtliches, ideales Ur-Athen imaginiert. Allein schon die Entgegensetzung von Beginn - Lenins Vorhersage, dass das 20. Jahrhundert von Kriegen und Revolutionen gekennzeichnet sein wird - und Ende müsste die Interpreten reizen, dem Spannungsbogen nach-denkend zu folgen. Und dann, so könnte man den Faden fortspinnen, wäre eben nicht mehr über Graecophilie und Weltflucht, politische Gegenwartsanalyse und Revolutionsgeschichte, Französische und Amerikanische Revolution zu diskutieren, sondern über Arendts Konstruktion, die dahin zurückführt, wo auch nach ihrer Meinung alles begann: in Athen - und zwar im Athen der Tragödie, also der Literatur! Und das ist doch für sich genommen revolutionär. ${ }^{19}$

Einen allerersten Anfang einer konsequenten Sammlung und dann Auswertung der Stellen, an denen Arendt auf Platon zurückgreift, macht jetzt Marie-Josée Lavallée (2019). Sie ist die erste überhaupt, die die Spezifik von Arendts Lektürepraxis bezogen auf antike Autoren analysiert. Die vielbeschworene Weimarer Prägung, die bei Weitem nicht auf Heidegger und Jaspers begrenzt werden kann, wird hier ebenso ansatzweise kontextualisiert wie die Freisetzung der Lektüren für die eigene systematische Arbeit. Insbesondere das Kapitel über den poiesis-Begriff (Lavalleé 2019, S. 233-285) legt offen, wie Arendt ihre Umformungen der antiken Konzepte vornimmt: indem sie sie aus ihren genuin philosophischen, das heißt letztlich genuin asozialen und antipluralistischen metaphysischen „Höhlen“ befreit und radikal ins Politische, also dem genuin sozialpluralistischen des Mensch-Seins übersetzt. Insofern ist es völlig falsch von der Athener Polis als „Modell“ zu sprechen. Sie ist viel mehr und zugleich viel weniger. Mehr, weil hierin ein idealtypisches Zusammenle-

\footnotetext{
18 „Nicht geboren zu sein, übertrifft/Jeden Begriff. Doch wann's erschien,/Ist das zweite weithin dies,/ Eilends zu gehen, von wann es kam“ (Arendt 2020, S. 419). Tatsächlich stammt die Übertragung von Emil Staiger. Arendt besaß Staigers Ausgabe. In Reinhardts Schriften konnte nirgends eine Übersetzung dieser Verse ausfindig gemacht werden. Der Vers 1144, der den Abschluss des Buches bildet, lautet bei Arendt: „,das Leben aufglänzen machte““, während Staiger (und viele andere ihm ähnlich) übersetzt: „Dem Leben einen höhern Glanz zu geben“".

${ }^{19}$ Der erste originelle, das heißt eigenständige Umgang mit Arendts vermeintlicher Graecophilie bietet Eva von Redecker (2020), in der die Autorin den Eigenständigkeitsehrgeiz und die Widerstandskraft betont, die Arendt in der Polis sah.
} 
ben imaginiert werden kann; weniger, weil dieser Idealtypus selbst zu einem Ideal wurde und in der Folge zu einer ungeschichtlichen platonischen Idee. Was sich in Lavallées Buch zumindest andeutungsweise verstehen lässt, ist die daraus folgende Dialektik zwischen der Anerkenntnis Arendts, dass jedwede politische Theorie nach dem Traditionsbruch einer Evaluierung bedarf und dass diese Theorien mit Platon und Aristoteles und den griechischen Dramatikern beginnen. Es ist jene Dialektik, die, wie bereits erwähnt, die Grundlage für Arendts Denken schafft.

Mit der grundlegenden Arbeit von Oliver Bruns (Bruns 2018) liegt nun erstmals eine ebenso genaue, wie sehr gut informierte Arbeit vor, die Arendts Beschäftigung mit antiken Autoren systematisch rekonstruiert und die Ergebnisse für eine ideengeschichtliche Spurensuche des Menschenrechtsdiskurses der „Alten“ nutzt. Insbesondere die für die „Entstehung des Politischen bei den Griechen“ (Christian Meier) und den Römern wesentliche Unterscheidung zwischen juridischen und moralischen Diskursen erhalten in Bruns' Deutung von Arendts Überlegungen wesentliche Klärungen.

In jedem Falle sind für die Forschung auf dem Feld Arendt/Antike noch große Lücken zu schließen, die möglicherweise durch komparative Studien, wie sie etwa Liisi Keedus über Arendt und Strauss (Keedus 2015) vorlegte, geschlossen werden können. Keedus' Arbeit koppelt den Vergleich an die Zeitläufte, also an die Weimarer Republik und den Nationalsozialismus und könnte insofern Vorbild für Studien sein, die Arendt etwa in den Kontext der Antikenrezeption verorten. Ähnlich verfährt Benjamin Aldes Wurgaft (Wurgaft 2015), der neben Strauss noch Levinas mit Arendt ins Verhältnis setzt. Weitaus sichtbarer, weil öffentlich ausgetragen, war die Auseinandersetzung zwischen Eric Voegelin und Arendt über die 1951 publizierte Totalitarismus-Studie. Ein Sammelband (Arendt und Voegelin 2015) vereint die Texte und Briefe der Kontroverse, die auf beiden Seiten die ,antike“ Struktur der Äußerungen offenlegen. Dass Arendt in Voegelin und seinem Aufbruchversuch einer „New Science of Politics“ einen grundsätzlich Verbündeten in der fundamentalen und ebenso fundamental notwendigen Neukonstituierung des Nachdenkens über den Menschen und sein Handeln in der Welt gesehen hat, kam rein äußerlich in ihrer Beteiligung an seiner Festschrift zum Ausdruck. Was dank der Gegenüberstellungen von Arendts Denken mit dem von Strauss und insbesondere Voegelin deutlich wird, ist, dass die Frage nach dem Status des antiken Denkens für die Gegenwart nicht lediglich Folge des humanistischen Gymnasiums und auch keine Form des Eskapismus sind. Vielmehr äußert sich in dem Rückgang eine Verantwortung gegenüber dem Gedachten per se, als genuine Selbstversicherung, dass die „Kette“ menschlichen Denkens und Handelns, zwar immer geschichtlich verortbar, zugleich davon immer unabhängig ist, weil sie auf die jeweilige Gegenwart bezogen werden kann.

Was diese drei ausgewählten Studien zudem belegen, ist etwas, das in vielfacher Hinsicht noch nicht ausgelotet ist. Nämlich die Wechselverhältnisse, die zwischen Arendt und anderen AutorInnen bestehen. Zwar ist die Fixierung auf Martin Heidegger, Karl Jaspers und Walter Benjamin, aber auch Gershom Scholem weitgehend überwunden, aber selbst bei Untersuchungen über sie und ihre Beziehung zu Arendt geht es ausgesprochen monolithisch zu, wie die ältere Studie etwa von Dana Villa 
(1996) belegt. ${ }^{20}$ Dass sie zu den Genannten zwar Aufsätze geschrieben hat, aber kein vollumfängliches Buch, anders als zu Augustinus und zu Rahel Varnhagen, lässt sich weder mit dem Hinweis auf die Emigration und die durch den Zweiten Weltkrieg und die Shoah gänzlich veränderten Koordinaten erklären, noch mit der vermeintlichen Fokussierung auf sogenannte systematische Fragestellungen. Arendts Konzept von Pluralität und Pluralisierung hingegen schließt ein, dass die Vielstimmigkeit des Lebens und Denkens sich im Denken selbst zu spiegeln habe. Es ist von daher müßig, sie einem „Lager“ zuzuordnen. Vielmehr wäre es weitaus ertragreicher, aufzuhellen, wie Arendt das aufreizende und verstörende Mischen in ihren Schriften handhabt und welche Funktionen das Mischen jeweils hat. Zumal dann, wenn, wie im Falle der Heidegger- und Benjamin-Essays, sich apologetische und polemisierende Elemente nicht trennen lassen.

\section{Arendt und die Literatur}

Was für die antike Philosophie stimmt, ist mit nur sehr wenige Ausnahmen auch für Arendts Auseinandersetzung mit den antiken Dramatikern und Erzähler richtig. Eine davon ist Esther Schröter. In ihrer Studie, vor allem im Abschnitt „Die verschiedenen Erzählweisen“ (Schröter 2014, S. 108-156) kommt sie gelegentlich auf Homer zu sprechen, um so die handlungstheoretischen Absichten von Arendts Literaturanalysen herausarbeiten zu können.

Denn auch wenn sich, wie die wohl wichtigste Arbeit über Arendts Verhältnis zur Dichtung von Anne Bertheau (Bertheau 2016) belegt, ein adäquates Verständnis von ihrem Verhältnis zu Welt, Menschen und Denken nur dann gewinnen lässt, wenn die Bedeutung und Funktion von Literatur genauer ausgeleuchtet wird, so fehlt auch dort immer der Einbezug zur antiken Tradition. Dies soll hier nur festgestellt werden, denn die Arbeit von Bertheau ist zu wichtig, um sie nicht selbst in den Blick zu nehmen. Denn die Autorin legt eine der methodisch und interpretatorisch genauesten Studien vor, die in den vergangenen Jahren zu Arendt erschienen sind. Die Sondierungen im ersten Teil changieren klar und klug zwischen Referat und Deutung. So vorbereitet, wird die Selbstständigkeit der jeweiligen Positionen Arendts in Abgrenzung zu Heidegger und Adorno betont, ein zumindest im deutschsprachigen Zusammenhang verständliches Manöver. Allerdings nimmt Arendt keine Mittelposition zwischen einer ontologischen und einer gesellschaftskritischen Funktionalisierung und Instrumentalisierung der Literatur ein. Nach Bertheau, die sich hier vom Mainstream der literaturwissenschaftlichen Arendt-Forschung in Nuancen absetzt, ist es die ethische Dimension, welche im Mittelpunkt der Textarbeit steht. Allerdings habe diese „Ethik“ eben keine ,heilende“ Rolle, vielmehr biete sie Möglichkeiten für menschliches Handeln an, die in der philosophischen Tradition wenig beachtet worden seien.

Ob die von Susannah Young-ah Gottlieb (Gottlieb 2007) herausgegebene, teilweise übersetzte und eingeleitete Sammlung von Texten Arendts zu Literature and

${ }_{20}$ Die beste derzeit verfügbare Analyse des Verhältnisses von Arendt und Heidegger hat Tatjana N. Tömmel (2013) vorgelegt. 
Culture zu einem größeren Interesse an Arendts Texten zu diesen Feldern beitragen wird, bleibt weiterhin abzuwarten. Bislang lassen sich in der englischsprachigen Literatur keine Anstrengungen erkennen, die zahlreichen und zum Teil sehr prominenten Freundschaften und Kooperationen mit Poeten aus den USA als Teil ihres Lebens und Werkes zu analysieren. Das ist angesichts der Allgegenwart Arendts in den dortigen Literatur- und Kulturwissenschaften überraschend. Umgekehrt verweisen nicht einmal die zahlreichen Biografien über W. H. Auden, Mary McCarthy oder Susan Sontag in irgendeiner besonderen Weise auf Arendt. Worin hier das Desinteresse begründet ist, ist schwer zu beurteilen. Ob sich ausgerechnet in den USA die Trennung von Literatur und Philosophie bzw. politischer Theorie fortsetzt, die lange die Situation in Deutschland bestimmte?

Fraglos aber sind es die USA, in denen die mit Abstand meisten Arbeiten zu Arendt erscheinen. Mit Richard H. Kings Arendt and America (King 2015) liegt erstmals eine Monografie vor, die minutiös ihren Lebens- und Denkweg von 1941-1975 verfolgt. Dazu zieht King, anders als prominente KonkurrentInnen wie etwa Seyla Benhabib (1996, 2010, 2018), nicht nur Archivmaterial heran, sondern setzt sich ohne Großthesenbedürfnis entlang der Chronologie mit Arendts Überlegungen auseinander. Die radikale Einseitigkeit Kings, er kennt nur und ausschließlich die auf Englischschreibende und auf amerikanische Kontexte zielende Arendt, lässt durchaus eine neue Sicht auf ihr Denken zu.

\section{Arendt und das Recht}

Wer hätte je gedacht, dass RechtsphilosophInnen im weitesten Sinne diejenigen sein werden, die Arendt als im wahrsten Sinne des Wortes universale Denkerin etablieren würden? Dass ein Text, ja eigentlich nur eine formalartige These, nämlich die bereits zitierte Rede vom „Recht, Rechte zu haben“ überhaupt Aufmerksamkeit erzielen würde, war nicht vorauszusehen. ${ }^{21}$ Wer sich im Dickicht der Untersuchungen, die die sprichwörtlichen Regalmeter füllen, noch zurückfinden will, der muss zum Sammelband Arendt and Law (Goldoni und McCorkindale 2017) greifen. In der hervorragenden Reihe „Philosophers and Law“ bei Routledge erschienen, bietet er 23, zum Teil selbst schon zum Gegenstand von Sekundärliteratur gewordene Aufsätze; etwa jene von Benhabib und Jacques Rancière, die Arendts weit über die Formel hinausgehendes Interesse am Recht und am antiken nomos dokumentieren. Denn dass die politischen Grundlegungsfragen immer auch Fragen des Rechts sind, exemplarisch nachzuweisen an den vom Recht Ausgeschlossenen, den Flüchtlingen, letztlich Staatenlosen, das war Arendt zunächst persönlich und dann während ihrer Beschäftigung mit dem modernen Antisemitismus während des Pariser Exils klargeworden. Auch wer diese historische Perspektive ein wenig vermisst: Goldoni und McCorkindale haben mit ihrer Zusammenstellung ganze Arbeit geleistet. Das

\footnotetext{
21 Ein unmittelbar vor Abschluss dieses Berichts erschienener wichtiger Sammelband: Ino Augsberg, Steffen Augsberg und Ludger Heidbrink (2020), zeigt an, wie Arendts Formel, rechtliche Tendenzen in modernen Gesellschaften besser zu verstehen und zugleich zu verdecken hilft. Hier sei insbesondere auf die Texte von Ino Augsberg und Franz Reimer verwiesen.
} 
heißt, dass einsichtig wird, inwiefern Arendt im Kontext von Fragen der Staatenlosigkeit und der Rechte von Flüchtlingen nicht aktualisiert werden muss, um sie als Gesprächspartnerin für die Probleme der Gegenwart gewinnen zu können, sondern als Zeitgenossin behandelt werden kann. Denn die von ihr gemachten Erfahrungen und das von ihr aufgearbeitete Material sind insofern nicht veraltet oder obsolet geworden, als sie von Arendt selbst ins Exemplarische übersetzt wurden.

Bemerkenswert ist bei der Einordnung von Arendts Überlegungen zum Recht, dass sie sowohl während des Pariser Exils als auch in ihren verschiedenen Funktionen innerhalb der JCR die innerjüdischen Diskussionen intensiv verfolgte, zumindest in New York die maßgeblichen Protagonisten zum Teil sogar als Kollegen hatte, doch in ihren Schriften keinerlei Gebrauch von deren Überlegungen machte. Einer von ihnen, Jacob Robinson, sollte später sogar einer ihrer ärgsten Feinde werden.

Wer von Arendt und Recht redet, darf also von Eichmann in Jerusalem nicht schweigen. ${ }^{22}$ Denn genau in dieser bis heute andauernden Auseinandersetzung um das vermeintliche oder tatsächliche Skandalbuch, war Robinson auf der Seite jener, die die vermeintliche ,Exkommunizierung“ (Amos Elon) aus der jüdischen Gemeinschaft betrieben haben. Exemplarisch seien zwei Publikationen herausgegriffen, die je eigene Zugänge bieten. Peter Burdon (Burdon 2018) liefert, auch in der Auseinandersetzung mit der sehr umfangreichen und kaum Gemeinsamkeiten aufweisenden Sekundärliteratur, den derzeit besten Überblick in Sachen Arendt/Eichmann. Burdon rekonstruiert systematisch sowohl den Aufbau des Buches, also auch der einzelnen historischen Ereignisse, wie sie im Prozess und in Eichmann in Jerusalem behandelt wurden. Somit werden die Leser in die Lage versetzt, Arendts Umgang mit beidem, Geschichte und Prozess, genauer zu verstehen. Dass Burdon Arendts Positionen, die alles andere als widerspruchsfrei sind, positiv reflektiert, bedeutet allerdings nicht, dass er sich in die alte Spannung zwischen ,,verdammen“ und ,begeistern“ begibt. Insofern zieht der in Australien Lehrende eine Konsequenz aus Dan Diners immer wieder hervorgehobenem Tatbestand, dass nicht „Eichmann in Jerusalem“ im Mittelpunkt der Kontroversen stehe, sondern die diversen Kontroversen um das Buch.

Es ist daher stimmig, dass Richard J. Golsan und Sarah M. Misemer (Golsan und Misemer 2017) den Titel The Trial that Never Ends für ihren Sammelband gewählt haben. Denn in der Tat: Fast scheint es, als müsse jede neue Generation das Buch, das zunächst in fünf, davon abweichenden Teilen in der Zeitschrift New Yorker erschien, neu lesen und deuten. Dieser Eindruck wird durch die neun Artikel, denen eine ausführliche Einleitung voraus geht, bestätigt. ${ }^{23}$ Was die AutorInnen zudem bestätigen ist, dass Arendts Buch über die historischen Zusammenhänge hinaus grundsätzliche Fragen nach Darstellung, Historiografie und, wie es Leora

\footnotetext{
${ }^{22}$ Es sei ausdrücklich vermerkt, dass das Buch, das wie kein anderes zu einem Überdenken von Arendts „Bericht“ herausfordert, nämlich Bettina Stangneths Eichmann vor Jerusalem. Das unbehelligte Leben eines Massenmörders (2011), noch immer nicht die Rolle in der deutschsprachigen Forschung spielt, die es verdient hätte.

${ }^{23}$ Die ursprünglich für das Hamburger Magazin SPIEGEL geschriebene Rezension des Auschwitz-Überlebenden und Historikers Joseph Wulff, die der Piper Verlag mit Unterstützung Arendts zu verhindern wusste, wurde von Anja Keith und Detlev Schöttker erstmals in der $F A Z$ am 20. Januar 2020, S. N3, veröffentlicht. Sie gehört zweifellos zu den schärfsten und genauesten der damaligen Kritiken.
} 
Bilsky tut, „Truth and Judgment“ stellt. Der Band kann vor allem deshalb empfohlen werden, weil die Beiträger jeweils Arendts Gesamtwerk im Blick haben, wenn sie ihren „Bericht“ analysieren. Nach dem inzwischen klassischen Band Hannah Arendt: Revisited. „Eichmann in Jerusalem “ und die Folgen, den Gary Smith im Jahr 2000 bei Suhrkamp herausgab, liegt zum zweiten Mal ein die wesentlichen Aspekte des Buches abdeckender Sammelband vor.

Für die Kantianerin Arendt gibt es einen unleugbaren Zusammenhang zwischen Recht und Moral, wobei sie diesen Zusammenhang erst nach dem Eichmann-Buch ausdrücklich thematisierte. Doch dass sie bereits bei der Arbeit an dem „Bericht“ - es existieren zu keinem anderen Buch auch nur annähernd so viele Manuskripte, Entwürfe etc., wie von diesem - an eine nachträgliche Erläuterung oder Rechtfertigung des Gesagten dachte, lässt sich in den Briefwechseln mit Jaspers oder Scholem ablesen. Hierbei muss es auch zu einer erneuten Auseinandersetzung mit Kant gekommen sein. Die von Ronald Beiners erstmals vorgelegte Edition jener Texte, die zum ungeschriebenen dritten Teil von Arendts fragmentarisch gebliebenen Werk „Life of the Mind“ gehören, ist seit dem Erscheinen 1992 ein Dauerbrenner in der Arendt-Literatur. René Torkler (Torkler 2015) hat Arendts Kant-Rezeption nicht bloß rekonstruiert und die Eigenwilligkeit ihrer genuin nicht transzendentalphilosophischen Deutung herausgearbeitet, sondern die Zentralstellung der Idee des „Gemeinsinns“ (,sensus communis“) für Arendts Hermeneutik plausibel dargelegt. Die in der dritten Kritik ,erweiterte Denkart“ bildet, das weist Torkler klar nach, die Grundlage für Arendts Pluralismus-Verständnis und somit auch, und dies nicht als geschichtsphilosophischer Nebeneffekt, für ihr Verständnis von „Tradition“.

\section{Schluss}

Der vorgelegte Überblick zu neuerer Literatur von und über Arendt kann angesichts der immensen Dynamiken in diesem Forschungsfeld naturgemäß nur eine Momentaufnahme bilden. Gleichwohl wird sich, wie bereits angedeutet, mit der kritischen Ausgabe von Arendts Schriften künftighin sehr vieles ändern (müssen). Denn neben den benannten Lücken in der Forschung wird die kritische Ausgabe uns zwingen, noch genauer zu prüfen, wie Arendt dachte, welche Arendt wir im Blick haben, wenn wir über sie schreiben und was politischen Denken bei ihr heißt. Eine neue, sehr spannende Phase der Arendt-Forschung hat gerade erst begonnen.

Funding Open Access funding enabled and organized by Projekt DEAL.

Open Access Dieser Artikel wird unter der Creative Commons Namensnennung 4.0 International Lizenz veröffentlicht, welche die Nutzung, Vervielfältigung, Bearbeitung, Verbreitung und Wiedergabe in jeglichem Medium und Format erlaubt, sofern Sie den/die ursprünglichen Autor(en) und die Quelle ordnungsgemäß nennen, einen Link zur Creative Commons Lizenz beifügen und angeben, ob Änderungen vorgenommen wurden.

Die in diesem Artikel enthaltenen Bilder und sonstiges Drittmaterial unterliegen ebenfalls der genannten Creative Commons Lizenz, sofern sich aus der Abbildungslegende nichts anderes ergibt. Sofern das betreffende Material nicht unter der genannten Creative Commons Lizenz steht und die betreffende Handlung nicht nach gesetzlichen Vorschriften erlaubt ist, ist für die oben aufgeführten Weiterverwendungen des Materials die Einwilligung des jeweiligen Rechteinhabers einzuholen. 
Weitere Details zur Lizenz entnehmen Sie bitte der Lizenzinformation auf http://creativecommons.org/ licenses/by/4.0/deed.de.

\section{Literatur}

Agamben, Giorgio. 2002. Homo sacer. Die Souveränität der Macht und das nackte Leben. Frankfurt/Main: Suhrkamp.

Arendt, Hannah. 1951. Burden of our Time. London: Secker \& Warburg.

Arendt, Hannah. 1955. Elemente und Ursprünge totaler Herrschaft. Frankfurt/Main: Europäische Verlagsanstalt.

Arendt, Hannah. 2017. „Wie ich einmal ohne Dich leben soll, vermag ich mir nicht vorstellen. “ Briefwechsel mit den Freundinnen Charlotte Beradt, Rose Feitelson, Hilde Fränkel, Anne Weil und Helen Wolff. München: Piper.

Arendt, Hannah. 2018a. Thinking Without a Banister. New York: Schocken Books.

Arendt, Hannah. 2018b. Modern Challenge to Tradition. Göttingen: Wallstein.

Arendt, Hannah. 2019a. Wir Juden. Schriften von 1932 bis 1966. München: Piper.

Arendt, Hannah. 2019b. Sechs Essays. Die verborgene Tradition. Göttingen: Wallstein

Arendt, Hannah. 2020. Über die Revolution. Mit einem Nachwort v. Jürgen Förster. Hg. v. Thomas Meyer. München: Piper.

Arendt, Hannah, und Martin Heidegger. 1998. Briefe 1925 bis 1975 und andere Zeugnisse. Hg. v. Ludz, Ursula. Frankfurt/Main: Klostermann.

Arendt, Hannah, und Dolf Sternberger. 2019. „Ich bin Dir halt ein bißchen zu revolutionär. “ Briefwechsel 1946 bis 1975. Hamburg: Rowohlt.

Arendt, Hannah, und Eric Voegelin. 2015. Disput über den Totalitarismus. Texte und Briefe. Göttingen: V\&R unipress.

Augsberg, Ino, Steffen Augsberg, und Ludger Heidbrink (Hrsg.). 2020. Recht auf Nicht-Recht. Rechtliche Reaktionen auf die Juridifizierung der Gesellschaft. Weilerswist: Velbrück.

Baehr, Peter, und Philip Walsh. 2016. The Anthem Companion to Hannah Arendt. London, New York: Anthem Press.

Benhabib, Seyla. 1996. The Reluctant Modernism of Hannah Arendt. Thousand Oaks: SAGE.

Benhabib, Seyla. 2010. Politics in Dark Times: Encounters with Hannah Arendt. Cambridge: Cambridge University Press.

Benhabib, Seyla. 2018. Exile, Statelessness, and Migration. Princeton: Princeton University Press.

Bernstein, Richard J. 2020. Denkerin der Stunde. Über Hannah Arendt. Berlin: Suhrkamp.

Bertheau, Anne. 2016. „Das Mädchen aus der Fremde“. Hannah Arendt und die Dichtung. Münster: Transcript.

Bluhm, Harald. 1995. Hannah Arendts Politische Theorie - Neuere Editionen und Interpretationen. Politische Vierteljahresschrift. 36(1):112-121.

Blücher, Heinrich. 2020. Versuche über den Nationalsozialismus. Hg. v. Ringo Rösener, Göttingen: Wallstein.

Boll, Monika, Dorlis Blume, und Raphael Gross. 2020. Hannah Arendt und das 20. Jahrhundert. München: Piper

Bruhns, Oliver. 2018. Antike Grundlagen der Entstehung moderner Menschenrechte. Freiburg: Verlag Karl Alber.

Brunkhorst, Hauke. 1999. Hannah Arendt. Eine Einführung. München: C.H.Beck.

Burdon, Peter D. 2018. Hannah Arendt: Legal Theory and the Eichmann Trial. Abingdon: Routledge.

DeGooyer, Stephanie, Alstair Hunt, Lida Maxwell, und Samuel Moyn. 2018. Vom Recht, Rechte zu haben. Hamburg: Hamburger Edition

Geulen, Eva. 2016. Giorgio Agamben zur Einführung. 3. Auflage. Hamburg: Junius.

Gines, Kathyrn T. 2014. Hannah Arendt and the Negro Question. Bloomington: Indiana University Press.

Goldoni, Marco, und Christopher McCorkindale (Hrsg.). 2017. Arendt and Law. London, New York: Routledge.

Golsan, Richard J., und Sarah M. Misemer (Hrsg.). 2017. The Trial That Never Ends. Toronto: University of Toronto Press.

Gottlieb, Susannah Young. 2007. Reflections on Literature and Culture. Stanford: Stanford University Press.

Gutschker, Thomas. 2002. Aristotelische Diskurse. Aristoteles in der politischen Philosophie des 20. Jahrhunderts. Stuttgart: J. B. Metzler Verlag. 
Hacke, Jens. 2020. Das Politische der Freundschaft. Zeitschrift für Ideengeschichte 14(1):123-127.

Heuer, Wolfgang. 1990. Hannah Arendt - Politische Wissenschaft aus dem Blickwinkel von Minderheiten. Politische Vierteljahresschrift 31(1):109-121.

Keedus, Liisi. 2015. Crisis of German Historicism. Hannah Arendt and Leo Strauss. Cambridge: Cambridge University Press.

King, Richard H. 2015. Arendt and America. Chicago: The University of Chicago Press.

Kohn, Jerome, und Ron Feldman (Hrsg.). 2007. Hannah Arendt. Jewish Writings. New York: Schocken Books.

Krimstein, Ken. 2018. The Three Escapes of Hannah Arendt. New York: Bloomsbury Publishing.

Lavallée, Marie-Josée. 2019. Lire Platon avec Hannah Arendt. Montreal: Presses de 1'Université de Montréal.

Ludz, Ursula. 2020. Hannah Arendts Denkwege 1951 bis 1955. Jahrbuch Politisches Denken 2018 28:55-80.

Maffeis, Stefania. 2018. Transnationale Philosophie. Hannah Arendt und die Zirkulationen des Politischen. Frankfurt am Main: Campus Verlag.

Meyer, Ahlrich. 2019. Wie Hannah Arendt versuchte, Karl Marx beizukommen. Bemerkungen anlässlich des Erscheinens der Arendt-Gesamtausgabe. Sozial.Geschichte Online. https://sozialgeschichteonline.org/2019/06/24/vorveroeffentlichung-ahlrich-meyer-wie-hannah-arendt-versuchte-karl-marxbeizukommen-bemerkungen-anlaesslich-des-erscheinens-der-arendt-gesamtausgabe. Zugegriffen: 20 Mär 2021.

Orozco, Teresa. 1999. Zur Renaissance des Unpolitischen. Arendt-Lektüren wiedergelesen. Berliner Debatte INITIAL 10:95-110.

Prinz, Alois. 2012. Hannah Arendt oder die Liebe zur Welt. Berlin: Insel Verlag.

Redecker, Eva von. 2020. Revolution für das Leben. Frankfurt/Main: S. Fischer.

Schmidt, Jana V. 2018. Arendt und die Folgen. Stuttgart: J.B. Metzler.

Schröter, Esther. 2014. Erzählen, (Lebens-)Geschichte im Werk Hannah Arendts. Münster: LIT Verlag.

Sigwart, Hans-Jörg. 2016. The Wandering Thought of Hannah Arendt. London: Palgrave Macmillan.

Stangneth, Bettina. 2011. Eichmann vor Jerusalem. Das unbehelligte Leben eines Massenmörders. Hamburg: Arche Verlag.

Straßenberger, Grit. 2015. Hannah Arendt zur Einfuihrung. Hamburg: Junius.

Taminaux, Jacques. 1992. La fille de Thrace et le penseur professionel. Arendt et Heidegger. Paris: Payot. Torkler, René. 2015. Philosophische Bildung und politische Urteilskraft. Freiburg: Verlag Karl Alber.

Tömmel, Tatjana N. 2013. Liebesbegriff bei Arendt und Heidegger. Berlin: Suhrkamp.

Villa, Dana. 1996. Arendt and Heidegger. The Faith of the Political. Princeton: Princeton University Press. Weißpflug. Maike. 2019. Die Kunst, politisch zu denken. Berlin: Matthes \& Seitz.

Wurgaft, Benjamin Aldes. 2015. Thinking in Public. Strauss, Arendt, Levinas. Philadelphia: University of Pennsylvania Press. 